\title{
Direitos Humanos, Esporte e Educação Física: desafios e perspectivas
}

https://doi.org/10.11606/issn.1981-4690.v35i3p113-123

\section{Resumo}

Apresentamosos desafiose perspectivas envolvendo Direitos Humanos, Esporteea EducaçãoFísica como agentes promotores da dignidade da pessoa humana.Valemo-nos dos principais documentos basilares do tema em questão e apontamos a necessidade do fortalecimento da educação em Direitos Humanos para viabilizar a construção de saberes capazes de desenvolver uma cultura de respeito aos DHelegitimara luta pelos direitos sociais. Chamamosa atenção para oatendimentoàs necessidades dediferentes populações, especialmente, àsqueapresentam oTranstorno do Desenvolvimento da Coordenação com destaque para as questões envolvendo sua etiologia, os instrumentos de identificação e diagnóstico e a intervenção. Nosso entendimento é de que o Esporte e a Educação Física Escolar figurem como eixos articuladores dos $\mathrm{DH}$, promovendo uma relação dialógica na construção de saberes plurais, heterogêneos eexperenciais ao longo da existência humana.

PalAVRAS-ChAVE: Direitos fundamentais; Cidadania; Dignidade humana; Educação inclusiva; Cultura corporal; Transtornos motores.

\section{Introdução}

Atualmente, a partir de uma perspectiva reflexiva, observamos uma preocupação envolvendo a Educação Física, o Esporte e os Direitos Humanos (DH), sobretudo na condição de estabelecer contribuiçôes nos processos pedagógicos e educacionais na promoção da dignidade da pessoa humana e das garantias dos direitos fundamentais. Trata-se de pensar num contexto em que os $\mathrm{DH}$ representam valores essenciais para o entendimento e a compreensão da importância do movimento corporal para a vida plena, considerando o sentido da universalidade, ou seja, de que os $\mathrm{DH}$ são direitos de todos, tendo a possibilidade de diminuir a distância entre uma sociedade excludente e uma sociedade inclusiva.

Nesse texto, produzido a partir da palestra proferida pelo primeiro autor, por ocasiāo do "Seminário de Direitos Humanos em Educaçáo Física e Esporte: desafios e perspectivas", promovido pelo Núcleo de Direitos Humanos da EEFE-USP, temos a intenção de apresentar desafios e perspectivas sobre as contribuiçóes do Esporte e da Educação Física na promoção da dignidade da pessoa humana, sobretudo numa sociedade marcada por violaçóes e desrespeitos aos avanços teóricos e normativos dos $\mathrm{DH}$. A formação de uma cultura de $\mathrm{DH}$ apresenta-se como possibilidade axiológica a ser vivenciada nas escolas e na vida cotidiana, tendo a apreensão de valores morais, éticos, estéticos e espirituais como fins educacionais no ensino e na pesquisa.

Nesse sentido, apresentamos aqui, os principais documentos que sustentam as açóes relacionadas aos DH; sugerimos também, algumas perguntas para suscitar o espírito crítico de nossos leitores; e, aprofundamos a temática, no que tange os $\mathrm{DH}$ na educação física, em relação aos desafios e perspectivas quanto ao conhecimento e atendimento às necessidades de diferentes populaçóes, no caso, especificamente, de indivíduos com Transtorno do Desenvolvimento da 
Coordenação (TDC).

Obviamente que não temos a pretensão de esgotar o assunto. Queremos sim, chamar sua atenção para a relevância do tema, ou seja, lançar luz a uma problemática recorrente no território brasileiro, e, principalmente, para as possíveis contribuiçóes de pesquisas científicas na promoção da dignidade da pessoa humana e das garantias dos direitos fundamentais, nas áreas de Educação Física e Esporte. Desejamos a todos uma boa leitura e, mais que isso, a aquisição de saberes (ou mesmo de dúvidas) que os conduzam a refletir e discutir sobre DH, Educação Física e Esporte.

\section{Direitos Humanos, Esporte e Educação Física}

Mas, o que seriam os DH? Trata-se de um sistema aberto que permite, assume e fortalece a coexistência pacífica de diversas culturas, crenças, práticas e organizaçôes sociais. Eles são a sociologia da vida contemporânea, uma vez que envolvem fatos, fenômenos, processos sociais e relaçóes, tais como, mentalidades, estados da mente, imagem, representaçóes, interesses e percepçóes ${ }^{1}$, que visam, acima de tudo, o reconhecimento da dignidade, da igualdade e dos direitos inalienáveis do ser humano. Eles incorporam conceitos de cidadania democrática, cidadania ativa e cidadania planetária inspirados em valores humanistas enraizados em princípios, tais como liberdade e equidade, confirmando sua universalidade, indivisibilidade e interdependência ${ }^{1}$.

Nesse contexto, temos documentos internacionais que reafirmam ser a educação um direito humano. A "Carta Internacional dos Direitos Humanos" é composta por três instrumentos: (1) Declaração Universal dos Direitos Humanos ${ }^{2}$; (2) Pacto Internacional dos Direitos Civis e Políticos ${ }^{3}$; e (3) Pacto Internacional dos Direitos Econômicos, Sociais e Culturais ${ }^{4}$. Esses instrumentos delinearam e delineiam os $\mathrm{DH}$, e nos permitem observar quatro movimentos principais: (1) Liberdade Civil e Política; (2) Igualdade Econômica, Cultural e Social; (3) Direitos Ambientais e Desenvolvimento Sustentável; e (4) colocar em prática os momentos anteriores - marcados pelas mudanças sociais correntes ${ }^{5}$. Nesse último, incluímos o Esporte e a Educação Física.

O Esporte tem forte relação com a "Educaçáo", o "Desenvolvimento" e a "Inclusão Social". Inclusive, organizaçóes como a Organização das Naçóes Unidas (ONU) e o Conselho Europeu (CE), creditam ao Esporte papel chave na promoção dos direitos fundamentais das pessoas como seres humanos e cidadãos. Dito de outra forma, o Esporte pode ter papel de protagonista tanto em relação aos "Direitos Humanos "quanto à "Dignidade Humana" .

Nesse sentido, é preciso reafirmar que sua vivência compreendida como um fenômeno que envolve o movimento humano é um constructo cultural baseado em concepçóes antropológicas e axiológicas do ser humano. O Esporte assim compreendido torna-se crucial na aprendizagem e no desenvolvimento do ser humano ao longo da vida. Ele não representa, exclusivamente, o potencial biológico e fisiológico dos indivíduos, mas vai além, pois é um conjunto de características sistêmicas e complexas que são relacional, social e moral, que emergem do compartilhamento da natureza humana 5 .

No mundo moderno o significado do Esporte está baseado na concordância de sua importância incondicional para as pessoas, o Estado e a sociedade em geral. Pelo prisma humanístico, o ator principal é o próprio ser humano, sua felicidade, sua saúde, sua liberdade, sua dignidade, seu desenvolvimento integral e harmonioso e a possibilidade de mostrar todas as suas habilidades ${ }^{7}$, de obter autocontrole, de expressar-se de forma criativa, de estruturar-se mentalmente, de construir virtudes como justiça e honestidade, e de enfrentar a vida como uma experiência de aprendizagem enaltecendo valores como coragem e humildade 5 .

Percebemos que, por um lado, há o discurso a favor do Esporte e sua utilização como ferramenta de promoção dos valores humanos, da educação, do desenvolvimento, da paz, da cooperação, da solidariedade, da tolerância, da inclusão social e da saúde ${ }^{5,7}$. Mas, por outro lado, o que nos se apresenta com maior frequência, é um Esporte, de rendimento, baseado na discriminação e na exclusão. Um Esporte que desrespeita e negligencia os $\mathrm{DH}$, que favorece a desigualdade social, oprimindo as crianças e adolescentes praticantes em suas singularidades e conflitos de valores.

Dessa forma, é crucial que fortaleçamos o Esporte como instrumento para a promoçáo dos DH. Para tanto, faz-se necessário conhecer a realidade, descrevê-la, problematizá-la e refletir sobre a mesma. Nesse sentido, suscitando reflexão mais focada sobre a relação entre Esporte e DH, apresentamos as questóes a seguir: (1) O Esporte expressa, verdadeiramente, a dignidade humana? (2) O Esporte é tratado como um Direito Humano? (3) O Esporte promove a inclusão social? (4) Como tornar o Esporte uma prática inclusiva? (5) É possível, por meio do Esporte, desenvolver valores de solidariedade, de cooperaçấo e ético-morais? 
(6) A prática do Esporte promove aprendizagem e desenvolvimento de habilidades ao longo da vida?

Discutir sobre o Esporte para a promoção dos $\mathrm{DH}$ no contexto educacional remete, necessariamente, ao contexto escolar. $\mathrm{Na}$ escola, esse patrimônio imaterial da Humanidade consta como um dos conteúdos da Educação Física Escolar, bem como de atividades extras, relacionadas a projetos esportivos.

O contexto escolar não é apenas produtor e reprodutor do conhecimento, mas é o "lócus" onde esse saber aparece sistematizado e codificado. Este é um espaço "social privilegiado" onde a ação pedagógica, a prática e a vivência dos $\mathrm{DH}$ são definidas. Nas sociedades contemporâneas, o contexto escolar é espaço de estruturação de concepçóes de mundo e da consciência social, de circulaçẫo e consolidação de valores, de promoçáo da diversidade cultural, da formação para a cidadania, de constituição dos sujeitos sociais e de desenvolvimento de práticas pedagógicas ${ }^{6}$. No contexto escolar a vivência dos $\mathrm{DH}$ deve estar garantida e mais do que isso, o Plano Nacional de Educação em Direitos Humanos ${ }^{6}$ (PNEDH) assume a educação em $\mathrm{DH}$ como um processo sistemático e multidimensional que pauta a formação do sujeito de direitos com base nas seguintes dimensões:

a) apreensão de conhecimentos historicamente construídos sobre direitos humanos e a sua relação com os contextos internacional, nacional e local;

b) afirmação de valores, atitudes e práticas sociais que expressem a cultura dos direitos humanos em todos os espaços da sociedade;

c) formação de uma consciência cidadã capaz de se fazer presente nos níveis cognitivo, social, ético e político;

d) desenvolvimento de processos metodológicos participativos e de construção coletiva, utilizando linguagens e materiais didáticos contextualizados;

e) fortalecimento de práticas individuais e sociais que gerem açôes e instrumentos em favor da promoção, da proteção e da defesa dos direitos humanos, bem como da reparaçáo das violaçôes.

Nesse documento, a educação desponta como um direito em si mesmo e caminho inequívoco para a obtenção de outros direitos. Ele, também, reforça a importância da educação ao ligá-la ao desenvolvimento humano e às suas potencialidades, destacando o respeito aos grupos socialmente excluídos $^{8}$. Vemos aqui, o papel fundamental do contexto escolar nas questóes relativas aos $\mathrm{DH}$.

Portanto, crianças e adolescentes não devem passar por situaçóes de exploração, discriminação, opressão, crueldade, violência e negligências às suas necessidades. Elas têm direito à liberdade - direito de ir e vir -; de opiniáo e expressão; de crença e culto religioso; de participar da vida comunitária sem discriminação; de divertir-se; de brincar e, destacamos aqui, de praticar esportes ${ }^{9}$. Assim, a prática do Esporte na escola, seja no contexto da Educação Física ou de atividades extracurriculares, deve zelar pela dignidade de crianças e adolescentes, livrando-os de tratamentos violentos, humilhantes e/ou desumanos.

Isso nos remete ao Estatuto da Criança e do Adolescente - ECA - (Lei 8069 de julho de 1990) ${ }^{10}$ que, advoga a favor da proteção integral à criança e ao adolescente e reforça o direito à educação, conferindolhe a responsabilidade pelo pleno desenvolvimento do indivíduo, ao mesmo tempo em que the assegura igualdade de condiçóes para o acesso e permanência na escola. E é nesse contexto que evidenciamos o direito de toda criança e todo adolescente a vivenciar o Esporte na escola. Por exemplo, não seriam os jogos escolares, locais e nacionais, verdadeiras oportunidades para fomentar as transformaçōes no esporte, transformações essas baseadas na Educação em DH? Nesse sentido, defendemos que as escolas públicas e particulares ocupam papel importantíssimo, em relacionar o Esporte aos DH.

Por sua vez, a Educação Física de qualidade é meio crucial para crianças e adolescentes adquirirem as habilidades, atitudes, valores, conhecimentos, a compreensão e apreciação relacionadas à cultura corporal, aspectos que são necessários para sua participação em atividade física e/ou no Esporte ao longo da vida e, na sociedade de forma geral ${ }^{11}$. Porém, nesses quesitos, alguns dados, deixando claros os desafios, nos convidam à reflexão.

No Brasil, 58,4\% das escolas de Educação Infantil náo possuem parque infantil; $63,5 \%$ das escolas do Ensino Fundamental Anos Iniciais; 57,3\% das escolas do Ensino Fundamental Anos Finais; e 42,1\% das escolas do Ensino Médio não possuem quadras cobertas $^{12}$. Além disso, as duas sessóes semanais de aulas de Educação Física correspondem a apenas 8\% do tempo escolar semanal, isto é, um percentual de horas insuficiente para a aprendizagem, desenvolvimento e aperfeiçoamento das habilidades relacionadas ao movimento, bem como para a adoção de hábitos de vida saudáveis.

Ainda, os dados indicam que nossas escolas se especializaram em ensinar o sentar, o ficar quieto. Nossas crianças e adolescentes passam, em média, 13 anos no ambiente escolar e saem especialistas no ato de sentar-se e, o que é pior, avessas ao movimento. A 
Organização Mundial da Saúde (ONU) revela que $39 \%$ da população latino-americana e caribenha são inativas, sendo que no Brasil a taxa de inativos é de, aproximadamente $47 \%$. O que gera um contrassenso e nos mostra o desconhecimento da importância do movimento em nossas vidas, como representado na afirmação que segue: "movimento é vida, e onde há vida há movimento constante"13.

Esses dados confirmam a negação do direito de todas as crianças e adolescentes a uma Educação Física de qualidade. Atualmente prepondera um modelo de estilo de vida que os ensina a serem buscadores de gratificaçóes imediatas, sedentários solitários, desconectados de si mesmos $^{14}$ e, acrescentamos, do próximo.

Assim, da mesma forma que para o Esporte, é crucial que fortaleçamos a Educação Física como instrumento para a promoção dos DH. Para tanto, faz-se necessário conhecer a realidade, descrevê-la, problematizá-la e refletir sobre a mesma. Nesse sentido, apresentamos as questôes a seguir: (1) A Educação Física Escolar é campo fértil para o desenvolvimento da Educação em Direitos Humanos? (2) A Educaçáo Física Escolar promove a participação igualitária de deficientes, grupos vulneráveis e outras minorias? (3) A Educação Física Escolar contribui para o empoderamento dos adolescentes, em especial, os do sexo feminino? (4) A Educação Física Escolar fomenta valores de solidariedade, de cooperação e ético-morais? (5) Como a formação do profissional de educação física pode contribuir para a Educação em Direitos Humanos?

Para finalizar este tópico, relacionamos os três temas tratados, ou seja, DH, Esporte e Educação Física, a partir da "Carta Internacional de Educação Física e Esportes"15, que foi um dos primeiros documentos internacionais a reconhecer a Educação Física e o Esporte como um direito de todos, especialmente, de crianças e adolescentes. No item 6 de seu preâmbulo afirma que a "Educação Física pode trazer diversos beneficios individuais e sociais, como a saúde, o desenvolvimento social e econômico, o empoderamento dos jovens, a reconciliação e a paz”. Esses dizeres reforçam o grande desafio de desenvolvermos açôes que garantam os DH no esporte e na educaçáo física, objetivando transformar nossa população em uma população mais ativa; fomentar a qualidade e a universalização do acesso às práticas da cultura do movimento humano.

No entanto, não basta reconhecer a Educaçáo Física e o Esporte como um direito de todos. Há a necessidade de ter conhecimentos para fundamentar a intervenção em Educação Física e no Esporte, de crianças e adolescentes que apresentam os mais diferentes perfis e necessidades. A 'mesma educação física', o mesmo direito, só serão alcançados mediante o reconhecimento e atendimento às demandas próprias das diferentes populaçóes e de seus indivíduos. Nesse sentido, a seguir trataremos, especificamente, dos $\mathrm{DH}$ no Esporte e na Educação Física, em relação aos desafios e perspectivas frente a indivíduos com Transtorno do Desenvolvimento da Coordenação (TDC).

\section{Direitos Humanos no Esporte e na Educação Física: desafios e perspectivas frente a indivíduos com TDC}

TDC é um transtorno crônico e comum, com prevalências estimadas entre $2 \%$ a $20 \%$ da população escolar ${ }^{16,17}$ que se dá no desenvolvimento e aprendizagem de habilidades motoras e que interfere negativamente nas atividades diárias, sociais e escolares ${ }^{17,18}$. É de natureza idiopática e ocorre independentemente da cultura, raça e condições socioeconômicas ${ }^{19}$.

Evidências sugerem que o TDC é um transtorno neurodesenvolvimental único e que pode co-ocorrer com um ou mais transtornos neurocomportamentais e neurodesenvolvimentais ${ }^{20}$. Comumente, estes transtornos incluem $\mathrm{ADHD}^{21}$, Transtorno do Desenvolvimento da Fala e Linguagem ${ }^{22}$, Dislexia ${ }^{23}$; Problemas afetivos-sociais ${ }^{24}$, entre outros. No entanto, o TDC tem sido negligenciado nas áreas da saúde e educação ${ }^{25,26}$.

As dificuldades no desempenho motor de indivíduos com TDC são frequentemente vistas como "menos graves" e, sendo assim, não garantem a mesma atenção das necessidades dos indivíduos com prejuízos mais severos nos movimentos, como os decorrentes da paralisia cerebral. Assim, argumenta-se que estabelecer uma rede de benefícios para avaliação e intervenção no TDC não se justifique como um investimento para a sociedade. Porém, os valores de prevalência - TDC é, de longe, o mais frequente transtorno motor com repercussão nas Atividades de Vida Diária (AVD) e Atividades de Vida Escolar (AVE) - e as descobertas sobre as consequências do TDC claramente sugerem que o TDC é um "fardo" considerável para o indivíduo e seus familiares e, portanto, ele merece atenção e investimento do ponto de vista da sociedade.

A influência do TDC sobre as AVD e AVE, e, secundariamente, sobre participação social, saúde física, e preocupaçóes com a saúde mental, combinada com altas taxas de prevalência indicam que o "fardo" social e econômico é considerável. Assim, para favorecer uma perspectiva de garantia dos $\mathrm{DH}$ de indivíduos com TDC, em relação ao direito de acesso a uma Educação 
Física de qualidade, ou seja, direito à 'mesma educaçáo física' ofertada aos indivíduos sem acometimento, faz-se necessário adquirir conhecimentos sobre o transtorno. Nessa linha, a seguir, dentre as muitas questóes que permeiam o TDC, destacaremos os estudos sobre: (1) Etiologia; (2) Instrumentos de identificação e diagnóstico; e (3) Diretrizes para a Intervenção.

\section{Etiologia do TDC}

A relação entre as estruturas cerebrais não é simples, no indivíduo com TDC essa conexão é ainda mais ambígua ${ }^{27}$. Os prejuízos cerebrais pré e perinatais são encontrados somente em um terço desses indivíduos ${ }^{28}$. Esses autores afirmam também que a grande parte dos indivíduos com TDC não possuem anomalias macroscópicas no cérebro, mas disfunçôes neurológicas com anormalidades nos neurotransmissores ou sistemas receptores.

As disfunçôes neurológicas, de uma forma geral, são classificadas em simples e complexas ${ }^{29}$ e o TDC pode estar, ou não, associado à forma simples ${ }^{27}$. Nesse caso, os fatores pré e perinatais, como: nascimento prematuro, retardo severo do crescimento intrauterino e asfixia perinatal, presumivelmente tem papel adicional.

Nos últimos 20 anos houve considerável quantidade de estudos sobre os mecanismos subjacentes ao TDC, os quais suscitaram diversas hipóteses envolvendo as estruturas cerebrais. Uma primeira hipótese, a do déficit de automatização indica que crianças com TDC apresentam dificuldades em realizar habilidades motoras automaticamente ${ }^{30}$. Já a hipótese do déficit de modelagem interna sugere dificuldade na formação de modelo interno, a partir do qual as consequências sensoriais esperadas - que permitem a comparaçáo entre o movimento desejado e o efetuado - são obtidas ${ }^{30-32}$.

Esses estudos têm utilizado técnicas de neuroimagem, como imagem de ressonância magnética funcional (IRMF); imagem de tensor de difusão (ITD); tomografia computadorizada com emissão de fótonúnico (TCEFU); e eletroencefalograma (EEG) como meios para identificar as vias e regióes de ativação envolvidas no processo sensoriomotor cortical ${ }^{33,34}$.

Estudos que utilizaram a IRMF, por exemplo, mostraram alteraçôes da organização microestrutural da matéria cinzenta, particularmente nos tractos sensório motores que incluem o tracto cortiço espinhal, o radial talâmico posterior, e sub-região parietal do corpo caloso $^{35,36}$. Outros resultados indicaram uma rede neural pobremente integrada envolvendo estruturas sensório-motoras ${ }^{37}$. Juntos, esses resultados oferecem suporte para a hipótese que crianças com TDC mostram diferenças na estrutura neural e funcional comparada com crianças de desenvolvimento típico. Nesse sentido, surge a necessidade de realização de estudos de natureza longitudinal que permitam confirmar ou não tal hipótese.

Em termos comportamentais, essas diferenças na estrutura neural e funcional podem afetar o planejamento antecipatório e a aprendizagem observacional, e reduzir a automatização da habilidade motora, promovendo grande confiança no controle baseado no feedback mais lento e estratégias compensatórias ${ }^{19}$. Essas descobertas podem ser incorporadas em uma abordagem multicomponentes do TDC que considera a interação entre o indivíduo, a tarefa e as restriçôes ambientais ${ }^{38,39}$.

Mas, apesar dos esforços, entendemos que a etiologia subjacente ao TDC permanece desconhecida. As evidências apontam, de forma geral, na direção de déficits sutis no sistema neurológico ${ }^{40}$. Mas, concordamos com BRown-Lu e ZWICKer ${ }^{34}$ que defendem a necessidade de realização de mais estudos envolvendo técnicas de imagem cerebral, buscando: (1) reforçar e ampliar o conhecimento sobre os mecanismos subjacentes ao TDC; (2) investigar preditores do TDC; e (3) verificar a efetividade e a mudança neoplástica associada à intervenção e à reabilitação de crianças e adolescentes com TDC.

\section{Identificação e Diagnóstico do TDC}

A identificação de crianças com transtornos motores deve ser realizada precocemente ${ }^{41,42}$. Entretanto, o diagnóstico formal do TDC, abaixo da idade de 5 anos, deverá ser feito somente em casos de prejuízos severos, dada a grande variabilidade no desenvolvimento motor ${ }^{19}$. Os autores recomendam que, nessa situação, a decisão para diagnosticar deva se pautar nos resultados de, pelo menos, duas avaliações realizadas com intervalo mínimo de três meses.

A identificação do TDC pode ser feita informalmente pelos pais, familiares e/ou professores ao perceberem que as crianças e ou adolescentes não alcançam os marcos motores (por exemplo, caminhar com equilíbrio), apresentam dificuldades em tarefas de autoajuda (por exemplo, abotoar camisa), tarefas escolares, nas relaçóes sociais, ou em tarefas motoras (por exemplo, escrever, conversar no recreio, pular corda). A função também pode ser avaliada por profissionais como, entre outros, o fisioterapeuta e o pediatra ${ }^{43}$. Assim, tanto a criança quanto o adolescente podem ser observados e avaliados informalmente em diferentes contextos: doméstico, escolar, de lazer e em consultórios ${ }^{44}$. 
A utilização de instrumentos, como escalas e questionários, considerados como "filtro" para identificação do TDC são fundamentais nesse processo, tanto pelo baixo custo quanto pela economia de tempo, mesmo diante da carência de estudos voltados para o processo de validação e refinamento desses instrumentos para tornar seus resultados mais confiáveis e válidos ${ }^{19}$.

Estudiosos têm se mostrado favoráveis ao uso de questionários respondidos por crianças ${ }^{41,45}$, professores $^{46,47}$ e por pais ${ }^{42}$. O Developmental Coordination Disorder Parent Questionnaire (DCDQ) ${ }^{48}$, sua versão revisada (DCDQ-R) ${ }^{49}$, e a Lista de Checagem do teste MABC-2 (LC) $)^{50,51}$ focam no nível de atividade individual (por exemplo, auto-cuidado, habilidades com bola) e contêm itens que referemse e relatam funçóes corporais subjacentes. Destes, o DCDQ/DCDQ-R tem passado por processos de validação mais frequentes na literatura. Por outro lado, a LC do teste MABC-2 tem recebido menos atenção ${ }^{19}$.

Portanto, o DCDQ-R parece, até o momento, ser o único questionário com um bom respaldo em termos de evidências que validem sua utilização. Embora, outros questionários e avaliaçóes - especialmente aqueles que usam observação direta das AVD, jogos, e outras atividades infantis - podem ser muito úteis e, portanto, devem ser examinados futuramente.

Por sua vez, a avaliação com norma referenciada compreende a aplicação de testes motores apropriados, válidos, confiáveis e padronizados. Devem ser aplicados por profissionais (entre eles, pediatras, fisioterapeutas, terapeutas, psicólogos, fonoaudiólogos, profissionais de educação física), envolvendo tanto medidas quantitativas quanto qualitativas ${ }^{43}$. Como as medidas qualitativas dependem de julgamento mais subjetivo do que as quantitativas ${ }^{52}$, recomenda-se que sejam usadas de forma complementar na identificação de crianças e adolescentes com $\mathrm{TDC}^{53}$.

Os testes motores que têm sido aplicados nesse processo são: (1) Movement Assessment Battery for Children (MABC - 1 e 2) $)^{50,51}$; (2) BruininksOseretsky Test Of Motor Proficiency (BOT - 1 e 2) $)^{54}$; (3) Köerperkoordinationstestfür kinder $(\mathrm{KTK})^{55}$; (4) Zurich Neuromotor Assessment Battery (ZNA) ${ }^{56}$; (5) The McCarron Assessment of Neuromuscular Dysfunction ${ }^{57}$; (6) The Peadbody Developmental Motor Scales - 2a ediçăo ${ }^{58}$; (7) The Bayley Sclaes of Infant Development - 3a ediçâo ${ }^{59}$; (8) The Zuk Assessment ${ }^{60}$; (9) The Test of Groos Motor Development- $2^{\mathrm{a}}$ edição ${ }^{61}$. No entanto, nos últimos dez anos, os testes motores mais utilizados têm sido o MABC-2 e o BOT-2.

A escrita é uma atividade crucial para crianças e adolescentes. Essa tarefa tem se mostrado com forte poder discriminativo em indivíduos com transtornos desenvolvimentais ${ }^{62}$. Mas, no caso de crianças com TDC, não se tem clareza se essa habilidade é um marco para a identificação do TDC ou se, em certos casos, é uma co-ocorrência ${ }^{19}$. Para avaliar essa habilidade temos os seguintes instrumentos: (1) The Handwriting Proficiency Screening Questionnaire ${ }^{63}$; (2) Concise Assessment Methods for Children's Handwriting ${ }^{64}$; (3) The Detailed Assessment of Speed of Handwriting ${ }^{65,66}$; (4) Minnesota Handwriting Assessment ${ }^{67}$; (5) The Diagnosis and Remediation of Handwriting problems ${ }^{68}$; (6) Children's Handwriting Evaluation Scale-Manuscript ${ }^{69}$; (7) Evaluation Tool of children's Handwriting ${ }^{70}$; (8) Test of Legible Handwriting ${ }^{71}$.

O processo de identificação e diagnóstico do TDC pode ser melhor conduzido a partir do conhecimento da existência de uma trajetória, ao longo dos anos, onde diversos comportamentos - cognitivos, afetivos, sociais e motores - são afetados e impactam negativamente tanto em tarefas do dia a dia quanto escolares, provocando atitudes agressivas ou tornando crianças e adolescentes excessivamente dependentes e passivos ${ }^{72}$. Assim, em suma, a identificação do TDC pode ser feita informalmente pelos pais, familiares e/ou professores ao perceberem atrasos e dificuldades motoras da criança. A partir da identificação, a criança ou adolescente deve ser encaminhado para uma avaliação com norma referenciada, ou seja, para realização de diagnóstico, que compreende a aplicação de testes motores apropriados.

\section{Diretrizes para a Intervenção}

A elaboração e o oferecimento de programas de educação física às crianças e adolescentes com TDC são açôes fundamentais para minimizar os prejuízos sentidos nos ambientes doméstico, comunitário e escolar ${ }^{73-75}$. Esses programas, quando apropriados às necessidades dessa população, são decisivos para recuperar e aproximar suas trajetórias desenvolvimentais às de seus pares de desenvolvimento típico ${ }^{19,76-78}$. Portanto, mesmo que haja impossibilidades que impliquem no atendimento aos critérios de identificação e diagnóstico do TDC, é aconselhável empreender ações de intervenção para auxiliar e melhorar a participação de crianças e adolescentes nas atividades desenvolvidas nos diversos ambientes que frequentam ${ }^{19}$.

No ambiente escolar, por exemplo, os transtornos motores podem afetar tanto as habilidades motoras finas, habilidades que estão presentes na maioria das atividades escolares, quanto as habilidades motoras grossas, presentes nas atividades da Educação Física e do Esporte, como jogos e brincadeiras e nas 
relaçóes sociais com seus pares ${ }^{19,79}$. Esses aspectos juntamente com as características e peculiaridades ambientais devem ser considerados no planejamento de programas de intervenção.

Planejar programas de intervenção não é uma tarefa simples, em função das características heterogêneas que acompanham o TDC. Por outro lado, temos fortes razóes para sua implementaçãa ${ }^{80}$, por exemplo, a persistência do TDC ao longo da infância, adolescência e fases desenvolvimentais posteriores; a co-ocorrência de outros transtornos desenvolvimentais e; os prejuízos à trajetória desenvolvimental são reais.

Os programas de intervenção, atualmente, são agrupados em três abordagens ${ }^{19}$ : (1) A abordagem orientada a estrutura e função corporal, abrange atividades que visam melhorar as funçóes corporais consideradas subjacentes à dificuldade motora funcional identificada; (2) $\mathrm{Na}$ abordagem orientada à atividade, o foco é melhorar o desempenho em uma atividade específica; e, (3), a abordagem, orientada a participação, visa melhorar a participação em dada atividade em situação da vida diária.

As abordagens orientadas à atividade e à participaçáo têm como conteúdo da intervenção a aprendizagem e o treinamento da habilidade ou de habilidades motoras específicas ${ }^{19}$. No caso dessas abordagens, as seguintes recomendaçóes são cruciais: (1) propor tarefas orientadas às necessidades da criança e ou adolescente, ou seja, propor atividades que tenham significado para eles; (2) propor tarefas orientadas à meta (com foco em atividades e na participação como descritos na versão infantil e adolescente da CIF; (3) escolher tarefa e contexto específico (o que é aprendido e em qual circunstância); (4) considerar que crianças e ou adolescentes têm papel ativo; (5) objetivar a funcionalidade, não a normalidade; e (6) promover o envolvimento ativo de pais e responsáveis favorecendo a transferência da aprendizagem ${ }^{19}$.

Os programas de intervenção bem elaborados ${ }^{81,82}$ são o marco inicial para a transformação de um ciclo de exclusão, autoexclusão e isolamento social vivenciado por crianças e adolescentes com TDC, nas atividades da Educação Física e do Esporte, bem como nas AVD e AVE, em uma trajetória desenvolvimental emoldurada pela autoconfiança, autocompetência, pelo sentimento de pertencimento, pela participação ativa nos diferentes ambientes (doméstico, comunitário, escolar), pela adoção de hábitos saudáveis e melhoria de sua qualidade de vida ${ }^{83}$.

\section{Considerações finais}

Há um grande desafio às práticas educativas relacionadas ao Esporte e à Educação Física frente aos $\mathrm{DH}$. Sobretudo no ensino, na pesquisa, nas vivências e aprendizagens dos $\mathrm{DH}$ enquanto possibilidades formativas na construção de uma consciência engajada em defesa dos direitos à vida, indicando condições para uma discussão pautada e mais ampla sobre os valores humanísticos, éticos-morais, educacionais e de saúde no tratamento dos problemas da área, tendo em vista a base crítica sobre a temática.

O conteúdo apresentado aponta um conjunto de direitos prescritos e teóricos que sustentam o debate sobre os $\mathrm{DH}$ na potencialidade de uma crítica reflexiva e, ao mesmo tempo, na busca por evidências científicas que possam viabilizar o enfrentamento do desafio posto. Neste sentido, entendemos que o movimento da dialeticidade no Esporte e na Educação Física, poderia possibilitar a construção de um saber capaz de desenvolver uma cultura de respeito aos $\mathrm{DH}$ e, ao mesmo tempo, poder ampliar e legitimar a luta pelos direitos sociais, entendendo o Esporte e a Educação Física como eixos articuladores dos $\mathrm{DH}$, caminhando numa relação dialógica na construção de saberes plurais, heterogêneos e experenciais ao longo da existência humana.

Mas, não podemos nos limitar a tratar de $\mathrm{DH}$ nas aulas de educaçáo física e nos esportes, pois o direito é uma necessidade concreta do ser humano em sua integralidade corpórea para realização da vida plena. É necessário conhecer sobre as reais condiçôes econômicas, históricas, motoras e culturais das pessoas em movimento, sobretudo entender a área de conhecimento, como direito humano. Entretanto, para que os conceitos contidos nos documentos normativos e na produção teórica sobre o valor da pessoa humana e o sentido da educação física escolar como direito humano é necessário entender sua complexidade a partir da realidade vulnerável que vivemos em relação aos $\mathrm{DH}$ na prática educativa, da realidade e necessidades de pessoas com características também atípicas. 


\begin{abstract}
Human rights, sport, and physical education: challenges and perspectives.

Wepresent thechallengesand perspectives involving Human Rights, SportandPhysical Educationasagents that promote human dignity. We use the main basic documents of the theme in question and point out the need to strengthen human rightseducation toenabletheconstruction of knowledgecapable of developing a culture of respect for Human Rights and legitimizing the struggle for social rights. We draw attention to meeting the needs of different populations, especially thosewith Developmental Coordination Disorder, highlighting issues involving its etiology, identification and diagnosis instruments, and intervention. Our understanding is that Sport and School Physical Education figure as articulating axes of Human Rights, promoting adialogical relationshipintheconstruction of plural, heterogeneousand experiential knowledge throughout human existence.
\end{abstract}

KEYWORDS: Fundamental rights; Citizenship; Human dignity; Inclusive education; Body culture; Motor disorders.

\title{
Referências
}

1. Voicu AV, Fuerea A, Visoin DF, et al. Practing Sports: a fundamental human right. Internl Sports Law Rev Pandektis. 2013;10:1-2.

2. United Nations. The United Nations Educational, Scientific and Cultural Organization. Universal Declaration of Human Rights: UNESCO, 1948.

3. United Nations. The United Nations Educational, Scientific and Cultural Organization. International Covenant on Civil and Political Rights: UNESCO, 1966.

4. United Nations. The United Nations Educational, Scientific and Cultural Organization. International Covenant on Economics, Social, and Cultural Rights: UNESCO, 1966.

5. Isidori E, Benetton M. Sport as Education: between dignity and Human Rights. Procedia Soc Behavl Sci. 2015;197: 686-693.

6. Shevchenko O. Role of Human Rights and decent work in Sports. Internl Sports Law Rev Pandektis. 2014;10:3-4.

7.Brasil. Plano Nacional de Educação em Direitos Humanos. Ministério dos Direitos Humanos. 3a impressão, 2018.

8. Fernandes AVM, Paludeto MC. Educação e Direitos Humanos: desafios para a escola contemporânea. Cad Cedes. 2010;30(81):233-249.

9. Donnelly P. Sport and Human rights. Sport Soc. 2008;11:381-394.

10. Brasil. Lei Federal n. 8.069, de 13 de julho de 1990. Estatuto da Criança e do Adolescente. Brasília: Senado Federal, 2005.

11. Popov S, Jakovlyev I. The Influence of physical exercise on improvement of cognitive function. TIMS Acta. 2017;11(2):110-120.

12. Brasil. Censo Escolar da Educação Básica 2016. Ministério da Educação, 2017.

13. Connolly KJ. The nature of motor skill development. J Human Mov Studies. 1977;3:128-143.

14. Mauerberg-Decastro E, Figueiredo GA, Campbell DF. The reality of inclusion in physical education in the Brazilian School System: facts, theories and practice. Rev Bras Educ Física. 2020;34:11-28.

15. United Nations. The United Nations Educational, Scientific and Cultural Organization. International Charter of Physical Education and Sport. Paris: United Nations Educational, Scientific and Cultural Organization, 1978.

16. American Psychiatric Association. Diagnostic and Statistical Manual of Mental Disorders, 5th edn. Arlington, VA: American Psychiatric Association, 2013.

17. Blank R, Smits-Engelsman BC, Polatajko H, et al. European Academy for Childhood Disability. European Academy for Childhood Disability (EACD): recommendations on the definition, diagnosis and intervention of developmental coordination disorder (long version). Dev Med Child Neurol. 2012;54:54-93.

18. World Health Organization. International Classification of Functioning, Disability and Health. Geneva: World Health Organization, 2001. 
19. Blank R, Barnett AL, Cairney J, et al. International clinical practice recommendations on the definition, diagnosis, assessment, intervention, and psychosocial aspects of developmental coordination disorder. Develop Med Child Neurol. 2019:61:242-285.

20. Missiúna C, Rivard L, Bartlett D. Early identification and risk management of children with developmental coordination disorder. Phys Occupational Ther Pediatrics. 2003;15:32-38.

21. Goulardins JB, Rigoli D, Licari M, et al. Attention déficit hyperactivity disorder and developmental coordination disorder: two separate disorders or do they share a common etiology. Behav Brain Res. 2015;292:484-492.

22. Hill EL. Non specific nature of specific language impairment: a review of the literature with regard do concomitant motor impairments. Int J Language Commun Dis. 2001;36:149-171.

23. Geuze RH, Kalverboer AF. Tapping a rhythm: a problem of timing for children who are clumsy and dyslexic. Adapted Phys Activity Quarterly. 1994;11:203-213.

24. Shoemaker MM, Kalverboer AF. Social and affective problems of children who are clumsy: how early do they begin? Adapted Phys Activity Quarterly. 1994;11:130-140.

25. Missiúna C, Moll S, Law M, et al. Mysteries and mazes: parents' experiences of children with developmental coordination disorder. Can J Occup Ther. 2006;73:7-17.

26. Wilson BN, Neil K, Kamps PH, et al. Awareness and knowledge of developmental co-ordination disorder among physicians, teachers and parents. Child Care Health Dev. 2013;39:296-300.

27. Hadders-Algra M. The neuronal group selection theory: a framework to explain variation in normal motor development. Develop Med Child Neurology. 2000;42:566-572.

28. Hadders-Algra M, Huisjes M, Touwen BCL. Preterm or small for gestacional age infants: neurological and behavioural development at the age six years. Eur J Pediatrics. 1988;147:460-467.

29. Touwen B. Variability and stererotypy in normal and deviant development. Clin Develop Med. 1978;67:99-110.

30. Zwicker JG, Missiuna C, Boyd LA. Neural correlates of developmental coordination disorder: a review of hypotheses. J Child Neurol. 2009;24:1273-1281.

31. Cantin N, Helene PJ, Thach TW, et al. Developmental coordination disorder: exploration of a cerebellar hypothesis. Human Movement Sci. 2007;26:491-509.

32. Wilson PH, Maruff P, Butson M, et al. Internal representation of movement in children with developmental coordination disorder: a mental rotation task. Develop Med Child Neurol. 2004;46:754-759.

33. Zwicker JG, Missiuna C, Harris SR, et al. Brain activation associated with motor skill practice in children with developmental coordination disorder: a pilot diffusion tensor imaging study. Pediatric Neurol. 2012;46:162-167.

34. Brown-Lu M, Zwicker JG. Brain imaging increases our understanding of developmental coordination disorder: a review of literature and future directions. Current Develop Dis Rep. 2015:2:131-140.

35. Debrabant J, Vingerhoets G, Van Waelvelde H, et al.. Brain connectomics of visual-motor deficits in children with developmental coordination disorder. J Pediatr. 2016;169:21-7.e2.

36. Zwicker JG, Missiuna C, Harris SR, et al. Developmental coordination disorder: a pilot diffusion tensor imaging study. Pediatr Neurol. 2012;46:162-7.

37. Caeyenberghs K, Taymans T, Wilson PH, et al. Neural signature of developmental coordination disorder in the structural connectome independent of comorbid autism. Dev Sci. 2016;19:599-612.

38. Newell K. Motor skill acquisition. Ann Rev Psychol. 1991;42:213-37.

39. Davids KW. The constraints-based approach to motor learning. In: Renshaw I, Davids KW, Savelsbergh GJP, editors. Motor learning in practice: a constraints led approach. London, UK: Routledge (Taylor \& Francis), 2010:3-16.

40. Licari MK, Billington J, Reid SL, et al. Cortical functioning in children with developmental coordination disorder: a motor overflow study. Exp Brain Res. 2015;233:1703-1710.

41. Cairney J, Veldhuizen S, Kurdyak P, et al. Evaluating the CSAPPA subscales as potential screening instruments for developmental coordination disorder. Arch Dis Child. 2007;92:987-91.

42. Schoemaker MM, Flapper B, Verheij NP, et al. Evaluation of the developmental coordination disorder questionnaire as screening instrument. Dev Med Child Neurol. 2006;48:668-73.

43. Ferreira LF, Freudenheim AM. Noçóes desenvolvimentais e o transtorno do desenvolvimento da coordenação. Curitiba: Editora CRV; 2017.

44. Sugden D, Wright HC. Motor coordination disorders. London: Sage: 1998.

45. Hay J, Hawes R, Faught BE. Evaluation of a screening instrument for developmental coordination disorder. J Adolesc

Health. 2004;34:308-13.

Rev Bras Educ Fís Esporte, (São Paulo) 2021 Jul-Set;35(3):113-123 • 121 
46. Junaid K, Harris SR, Fulmer KA, et al. Teachers' use of the MABC checklist to identify children with motor coordination difficulties. Pediatr Phys Ther. 2000;12:158-63.

47. Wright HC, Sugden DA. A two-step procedure for the identification of children with developmental co-ordination disorder in Singapore. Dev Med Child Neurol. 1996;38:1099-105.

48. Wilson BN, Kaplan BJ, Crawford SG, et al. Reliability and validity of a parent questionnaire on childhood motor skills. Am J Occup Ther. 2000;54:484-93.

49. Wilson BN, Crawford SG, Green D, et al. Psychometric properties of the revised developmental coordination disorder questionnaire. Phys Occup Ther Pediat. 2009;29:182-202.

50. Henderson SE, Sugden DA. Movement assessment battery for children: manual. London, UK: Psychological Corporation, 1992.

51. Henderson SE, Sugden DA, Barnett AL. Movement assessment battery for children. 2nd edn. London, UK: Harcourt Assessment, 2007.

52. Keogh JF, Sugden DA, Reynard DL, et al. Identification of clumsy children: comparison and comments. J Hum Mov Studies. 1979;5:32-41.

53. Henderson SE. The assessment of "clumsy" children: old and new approaches. J Child Psychol Psychiatry. 1987;28:511527.

54.Bruininks RH. Bruininks-Oseretsky Test of motor proficiency. circle pines, MN: American Guidance Service, 1978.

55. Kiphard EJ, Schilling F. KTK, Korperkoordinationstest fur Kinder Hogrefe, 2007.

56. Largo RH, Rousson V, Caflisch J, et al. Zurich Neuromotor Assessment. Zurich: AWE Verlag, 2007.

57. McCarron LT. McCarron assessment of neuro muscular development: fine and gross motor abilities. Dallas, TX: Common Market Press, 1997.

58. Folio MR, Fewell RR. Peabody developmental motor scales. 2nd ed (PDMS-2). Austin, TX: Pro-Ed, 2000.

59. Bayley N. Bayley scales of infant and toddler development: administration Manual. 3rd ed. San Antonio, TX: Psychological Corporation, 2006.

60. Zuk L, Tlumek H, Katz-Leurer M, et al. A new tool for identifying children with motor problems reliability and validity study. J Child Neurol. 2014;29:592-8.

61. Ulrich DA. Test of gross motor development-2 (TGMD-2). Austin, TX: ProEd, 2000.

62. Dhall JK. Handwriting-based model for identification of developmental disorders among North Indian children. School Psychol Int. 2015;37:51-63.

63. Rosenblum S. Development, reliability, and validity of the handwriting proficiency screening questionnaire (HPSQ). Am J Occup Ther. 2008;62:298-307.

64. Hamstra-Bletz L, De Bie J, Den Brinker B. Beknopte beoordelingsmethode voor kinderhandschriften: experimentele versie [Concise Evaluation Scale for Children's Handwriting: Experimental Version]. Lisse, Netherlands: Swets \& Zeitlinger, 1987.

65. Barnett AL, Henderson SE, Scheib B, et al. Detailed assessment of speed of handwriting (DASH). Boston, MA: Pearson, 2007.

66. Barnett AL. Motor assessment in developmental coordination disorder: from identification to intervention. Int J Disabil Dev Educ. 2008;55:113-29.

67. Reisman J. Minnesota handwriting assessment manual. San Antonio, TX: Harcourt Assessment, 1999.

68. Stott D, Moyes FA, Henderson SE. Diagnosis and remediation of handwriting problems. Cardiff, UK: DRAKE Educational Associates, 1985.

69. Phelps J, Stempel L. Children's handwriting evaluation scale for manuscript writing. Dallas, TX: Texas Scottish Rite Hospital for Cripples Children, 1987.

70. Amundson SJ. Evaluation Tool of Children's Handwriting: A. Homer, OT Kids, 1995.

71. Larsen S, Hammill D. Test of legible handwriting. Austin, TX: PRO-ED, 1989.

72. Missiúna C, Rivard L, Pollock N. Tradução: Magalhães LC. Revisão: Ribeiro J. Crianças com transtorno do desenvolvimento da coordenação: em casa, na sala de aula e na comunidade. Belo Horizonte: UFMG; 2011.

73. Cairney J, Hay J, Mandigo JJ, et al. Developmental coordination disorder and reported enjoyment of physical education in children. Eur Phys Educ Rev. 2007;13:81-98.

74. Cantell MH, Smyth MM, Ahonen TP. Clumsiness in adolescence: educational, motor, and social outcomes of motor delay detected at 5 years. Adapted Phys Activity Quarterly. 1994;11:115-129.

75. Zwicker JG, Harris SR, Klassen AF. Quality of life domains affected in children with developmental coordination 
disorder: a systematic review. Child Care Health Develop. 2013;39:562-580.

76. Hillier S. Intervention for children with developmental coordination disorder: a systematic review. Internet J Allied Health Sci Pract. 2007;5:1-11.

77. Wann J. Current approaches to intervention in children with developmental coordination disorder. Develop Med Child Neurol. 2007;19:405-415.

78. Smits-Engelsmann BCM, Blank R, Van Der Kaay AC, et al. Efficacy of interventions to improve motor performance in children with developmental coordination disorder: a combined systematic review and meta-analysis. Develop Med Child Neurol. 2013; 55: 229-237.

79. Smits-Engelsman BCM, Vinçon S, Blank R, et al. Evaluating the evidence for motor-based intervention in developmental coordination disorder: a systematic review and meta-analysis. Res Develop Dis. 2018;74:72-102.

80. Sugden DA, Chambers ME. Children with developmental coordination disorder. London: Whurr; 2005.

81. Hung WMY, Pang MYC. Effects of group-based versus individual-based exercise training on motor performance in children with developmental coordination disorder: a randomized controlled pilot study. J Rehab Med. 2010; 42:122-128. 82. Noordstar JJ, Van der Net J, Voerman L, et al. The effect of an integrated perceived competence and motor intervention in children with developmental coordination disorder. Res Develop Dis. 2017;60:162-175.

83. Missiúna C, Rivard L, Bartlett D. Exploring assessment tools and the target of intervention for children with developmental coordination disorder. 2006. Phys Occup Ther Pediatrics. 2006;26:77-89.

\begin{tabular}{r|r} 
ENDEREçO & \\
Lúcio Fernandes Ferreira & \\
Rua Pe. Monteiro de Noronha, 575 - Casa 64 & Submetido: 01/05/2021 \\
69028-140 - Manaus - AM - Brasil & Aceito: 02/06/2021 \\
E-mails: alucciofer@ufam.edu.br & \\
lucciofer@gmail.com &
\end{tabular}

